ARTICLE

https://doi.org/10.1038/s41467-019-08929-0

\title{
Continuous wave amplified spontaneous emission in phase-stable lead halide perovskites
}

Philipp Brenner ${ }^{1}$, Ofer Bar-On², Marius Jakoby ${ }^{3}$, Isabel Allegro ${ }^{1}$, Bryce S. Richards ${ }^{1,3}$, Ulrich W. Paetzold ${ }^{1,3}$, Ian A. Howard (1) ${ }^{1,3}$, Jacob Scheuer ${ }^{2}$ \& Uli Lemmer ${ }^{1,3}$

Sustained stimulated emission under continuous-wave (CW) excitation is a prerequisite for new semiconductor materials being developed for laser gain media. Although hybrid organicinorganic lead-halide perovskites have attracted much attention as optical gain media, the demonstration of room-temperature $\mathrm{CW}$ lasing has still not been realized. Here, we present a critical step towards this goal by demonstrating CW amplified spontaneous emission (ASE) in a phase-stable perovskite at temperatures up to $120 \mathrm{~K}$. The phase-stable perovskite maintains its room-temperature phase while undergoing cryogenic cooling and can potentially support CW lasing also at higher temperatures. We find the threshold level for CW ASE to be $387 \mathrm{~W} \mathrm{~cm}^{-2}$ at $80 \mathrm{~K}$. These results indicate that easily-fabricated single-phase perovskite thin films can sustain CW stimulated emission, potential at higher temperatures as well, by further optimization of the material quality in order to extend the carrier lifetimes.

\footnotetext{
${ }^{1}$ Light Technology Institute, Karlsruhe Institute of Technology, Kaiserstraße 12, 76131 Karlsruhe, Germany. ${ }^{2}$ Department of Physical Electronics, Tel-Aviv University, Ramat-Aviv, 6997 Tel-Aviv, Israel. ${ }^{3}$ Institute of Microstructure Technology, Karlsruhe Institute of Technology, Hermann-von-Helmholtz-Platz 1, 76344 Eggenstein-Leopoldshafen, Germany. Correspondence and requests for materials should be addressed to U.L. (email: uli.lemmer@kit.edu)
} 
R ecently, solution-processed metal-halide perovskite semiconductors have started to challenge the performance of traditional inorganic semiconductors in the field of thinfilm photovoltaics ${ }^{1,2}$. These perovskites possess the chemical structure $\mathrm{ABX}_{3}$, where $\mathrm{A}$ is an organic or inorganic cation, such as methylammonium $\left(\mathrm{CH}_{3} \mathrm{NH}_{3} / \mathrm{MA}\right)$, formamidinium $(\mathrm{CH}$ $\left.\left(\mathrm{CH}_{2}\right)_{2}\right) / \mathrm{FA}$ ) or cesium (Cs), $\mathrm{B}$ is a metal (commonly $\mathrm{Pb}$ ), and $\mathrm{X}$ is a halide $(\mathrm{Cl}, \mathrm{Br}$, or $\mathrm{I})$. With an impressive tolerance to grain boundaries and defects, perovskite thin films exhibit high charge carrier mobilities, as well as excellent absorption and emission properties $^{3}$. These properties of thin films of perovskite deposited, e.g., by spin-coating, can be surprisingly close to the performance of perovskite single crystals ${ }^{4}$. As a direct bandgap semiconductor, perovskites are well-suited not only for solar cells, but also for light-emitting devices ${ }^{5,6}$. Perovskites can be integrated in lightemitting diodes (LEDs) as the emitting semiconductor layer ${ }^{6-9}$, or applied in the form of nanocrystals for color conversion with InGaN-based LEDs as a potential replacement for traditional phosphors ${ }^{10}$. Of most relevance to the current work is the ability of perovskites to exhibit stimulated emission and serve as optical gain media in the near infrared and across the visible range ${ }^{11,12}$. The progress in perovskite lasers has been summarized in several recent reviews ${ }^{6,13-18}$. Low-threshold lasing under pulsed optical excitation has been demonstrated using various resonator geometries, including at wavelengths within the "green gap"19,20, where conventional III-V-semiconductors suffer from low efficiencies $^{21-23}$. However, to become commercially relevant (ultimately in an electrically pumped configuration), any novel gain material (such as perovskites) should demonstrate the ability to maintain optical gain under continuous wave (CW) operation and ideally at room temperature, or at least at temperatures accessible by Peltier cooling ( $>220 \mathrm{~K})$.

Obtaining $\mathrm{CW}$ operation has proven to be a major challenge for most solution-processable semiconductors, such as organic semiconductors and colloidal semiconductor nanocrystals. While the former class of materials has never demonstrated CW lasing, the latter only recently made this step, almost two decades after the initial investigations ${ }^{24-27}$. Despite this impressive achievement, further progress towards electrically pumped lasing is expected to be highly challenging for colloidal semiconductor nanocrystal devices due to their limited electrical transport characteristics.

Demonstrating CW lasing in thin perovskite films is of great interest, as this would present a solution-processable gain material exhibiting excellent electrical transport properties. However, to date CW stimulated emission has not been demonstrated in technologically relevant single-phase thin films. First demonstrations of CW lasing in perovskites were made in nanowires, where lasing was attributed to a condensate of polaritons enabled by the strong light-matter interaction between the photons and the cavity excitons ${ }^{28,29}$. True CW photon lasing in thin films, which is more relevant for practical applications, has proven to be difficult to achieve with the perovskite workhorse material methylammonium lead triiodide $\left(\mathrm{CH}_{3} \mathrm{NH}_{3} \mathrm{PbI}_{3}\right)$. This difficulty has been ascribed to the phase instabilities of the crystal lattice with temperature, the limited thermal conductivity, screening effects and/or the rotational freedom of the methylammonium cation ${ }^{30-32}$. Clear proof of $\mathrm{CW}$ lasing in $\mathrm{CH}_{3} \mathrm{NH}_{3} \mathrm{PbI}_{3}$ was demonstrated by Jia et al. at a very specific temperature of $100 \mathrm{~K}$, where different crystalline phases coexist upon optical pumping ${ }^{32}$. The requirement of the simultaneous presence of two crystal phases limited CW lasing to these special conditions, whereas at other temperatures, photon lasing has only been possible at pulsed excitation ${ }^{32}$. In this sense, although this previous demonstration of CW lasing was an impressive achievement, the need for the mixed-phase structure does not establish the ability of conventional, singlephase, perovskites to serve as gain material of sufficient quality for CW lasing.

Here, we demonstrate sustained CW lasing in a mixed cation system at the A site of the perovskite crystal structure. We use a mixed cation perovskite as it undergoes no phase change during the transition from room temperature to cryogenic temperatures. Consequently, we show that this phase-stable mixed-cationic perovskite can support CW optical gain at any temperature up to $120 \mathrm{~K}$. These findings are a significant step towards practical perovskite CW lasers operating at room temperature, which opens new avenues to the development of perovskite semiconductor laser diodes and the solution of the green gap problem.

\section{Results}

Temperature-dependent amplified spontaneous emission (ASE) under pulsed excitation. In our study we employ a triple cation system with a non-stoichiometric ratio of $\mathrm{Cs}_{0.1}\left(\mathrm{MA}_{0.17^{-}}\right.$ $\left.\mathrm{FA}_{0.83}\right)_{0.9} \mathrm{~Pb}_{0.84}\left(\mathrm{I}_{0.84} \mathrm{Br}_{0.16}\right)_{2.68}$ in the precursor solution as reported previously in order to obtain low optical gain thresholds and an improved stability ${ }^{12}$. The partial replacement of the MA cation results in an identical crystal phase for arbitrary iodine bromine ratios at room temperature. No phase transitions are known at lower temperatures 33,34 , or have been observed in the current work. The perovskite layers were prepared on sapphire substrates and post treated by an imprinting step to reduce the surface roughness and to improve the morphology ${ }^{35-37}$ (see Supplementary Figure 1 for atomic force microscopy images of both pristine and imprinted layers). The smaller roughness decreases the waveguide scattering losses and is, therefore, expected to reduce the ASE threshold ${ }^{12,35,38}$. ASE, a type of mirrorless lasing $^{39}$, is highly suited for studying the material's gain characteristics by eliminating the influences of the laser resonator (which can induce a strong temperature dependence through the thermo-optic coefficient of the material). Additionally, this approach eliminates any ambiguity that may arise from cavity effects, such as angular-dependent diffraction of certain wavelengths from gratings or spectral filtering from DBR mirrors, which can change the detected shape of the spontaneous emission spectra even without stimulated emission being involved. Accurately establishing the existence of lasing is indeed not experimentally trivial and for more information on the experimental verification of real lasing the reader is referred to the commentary by Samuel et al. ${ }^{40}$.

Figure 1 depicts the temperature-dependent ASE thresholds measured under $0.8 \mathrm{~ns}$ pulsed excitation. An example of the evolution of the emission spectra from a $185 \mathrm{~nm}$-thick perovskite film over a range of pump fluences at room temperature is shown in Fig. 1a. The film was mounted inside a cryostat (Janis STVP100, see Supplementary Figure 2 for a schematic of the experimental setup) allowing the intensity-dependence of the emission spectrum to be measured as a function of temperature. In order to unambiguously identify ASE, it is important to monitor the broadband spectra below threshold (the spontaneous emission (PL), PL refers to the spectral part not containing stimulated emitted photons), as well as the narrowband spectra above threshold. The change of the luminescence profile below and above threshold distinguishes the onset of ASE from artifacts in thin films, such as a spectral modification of the detected PL due to photon propagation in a waveguide close to cut-off conditions. At low excitation fluences, the PL spectrum can be clearly identified by a broadband emission profile with a full width at half maximum (FWHM) of $\sim 40 \mathrm{~nm}$ and a nearly Gaussian shape. As the pump fluence increases, an intense emission shoulder between 773 and $785 \mathrm{~nm}$ (superimposed onto 


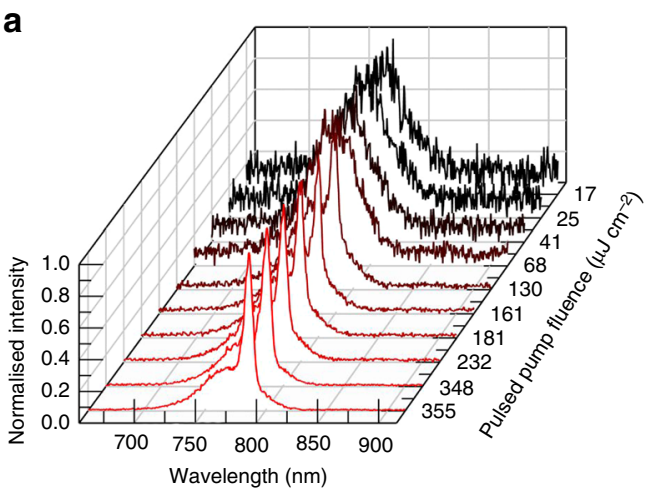

b
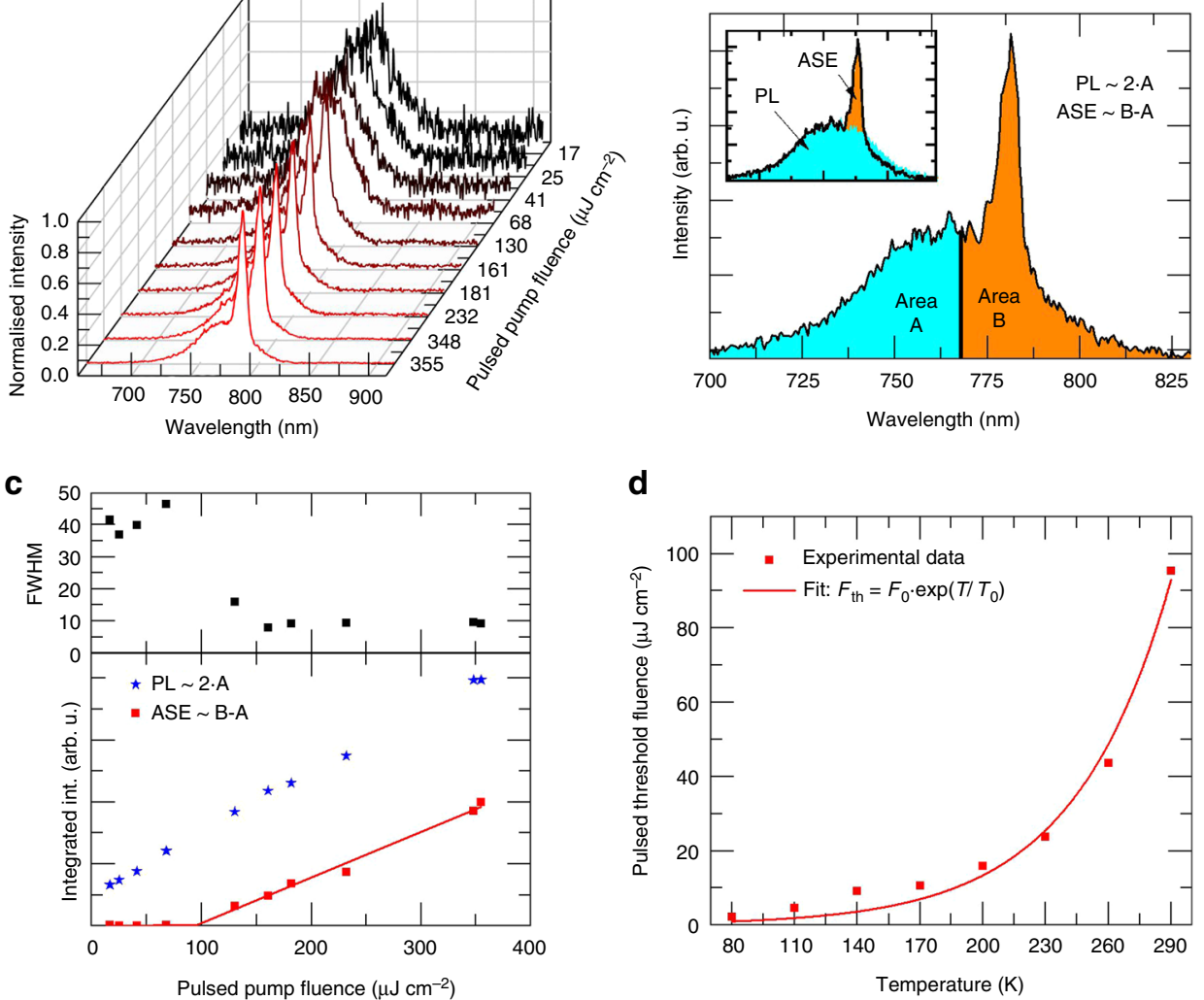

d

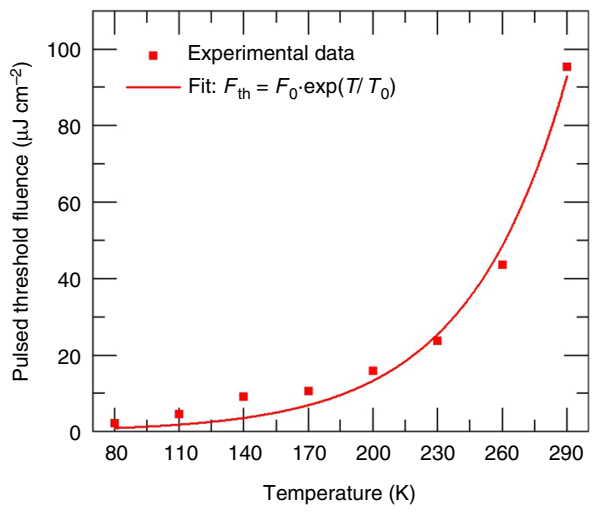

Fig. 1 Amplified spontaneous emission properties under pulsed excitation ( $0.8 \mathrm{~ns})$. a Example of data for room temperature showing the evolution of the emission spectra from which the amplified spontaneous emission (ASE) threshold is established. Different colors for the spectra are chosen for better distinguishability. $\mathbf{b}$ Regions of the spectrum used to determine spontaneous emission (PL) and ASE contributions. The inset graphically shows the assigned PL contribution (blue, area A and area A mirrored at the center wavelength) and ASE contribution (orange, area B-area A) and the real spectrum (black line). c PL, ASE, and full width at half maximum (FWHM) as a function of the pulse fluence. d ASE threshold as a function of temperature. The threshold decreases by more than an order of magnitude from room temperature to $80 \mathrm{~K}$. The red line is a fit to the empirical exponential threshold law typically observed in inorganic semiconductors. The characteristic temperature $T_{0}$ is found to be $46 \mathrm{~K}$

the PL spectra) emerges, indicating the onset of ASE. There are different methods to extract the threshold value for $\mathrm{ASE}^{41}$, here we follow the approach suggested by Sutherland et al. ${ }^{42}$. In the transition region from "predominant PL" to "predominant ASE", the spectrum consists of a superposition of contributions from both processes. The PL contribution is proportional to the integral of the emission in the higher energy half of the PLspectrum, which does not contain the ASE part $(680-767 \mathrm{~nm}$, area A in Fig. 1b). The ASE contribution can be estimated by integrating the low-energy half of the spectrum (area B in Fig. 1b) and subtracting the PL (area A) as illustrated in Fig. 1b. The integration boarder is chosen as the center wavelength of a Gaussian Fit to the spectra recorded at the lowest fluence (Supplementary Figure 3). Figure 1c depicts the total integrated PL intensity (approximated by twice the area A), the integrated ASE intensity (approximated by the difference between area $B$ and area A) and the narrowing of the FWHM as a function of the pump fluence. The threshold level is determined by the intersection of a linear fit of the ASE contribution with the pump fluence axis, yielding a threshold level of $95.3 \mu \mathrm{J} \mathrm{cm}^{-2}$. This value agrees well with the values reported in the literature for perovskite semiconductors ${ }^{6,15,16}$. Figure $1 \mathrm{~d}$ depicts the dependence of the threshold levels on the temperature. As the temperature is reduced from 290 to $80 \mathrm{~K}$, the ASE threshold decreases down to $.2 \mu \mathrm{cm}^{-2}$. It is empirically well-known that temperature-dependent current threshold in semiconductor diode lasers follows an exponential law ${ }^{43}$. Figure $1 \mathrm{~d}$ shows a fit of the pulsed threshold fluence using the following formula:

$$
F_{\mathrm{th}}=F_{0} \cdot \exp \left(\frac{T}{T_{0}}\right)
$$

where $T$ is the temperature, $F_{0}$ the threshold when $T$ approaches zero and $T_{0}$ is the characteristic temperature. As can be seen, there is a good agreement between the measurements and the fit, yielding a characteristic temperature of $T_{0}=46 \mathrm{~K}$. We note that this characteristic temperature is lower than that of most inorganic semiconductor laser diodes, implying a stronger dependence of the threshold levels of our perovskites on the temperature ${ }^{43}$. The reduction of the thresholds at lower temperatures can be attributed mainly to two distinct mechanisms. First, the temperature dependence of the carrier distribution in the conduction and valence bands leads to lower threshold values for achieving population inversion at lower temperatures. However, this effect by itself yields a temperature dependence that scales as $T^{\frac{3}{2}}$ (see Supplementary Note 1), indicating the impact of additional mechanisms. Second, the recombination lifetime increases for lower temperatures, leading to larger carrier densities (at a given excitation intensity) at lower temperatures. Consequently, the threshold density level is obtained at lower pump fluences when the temperature is low. The dependence of the carrier lifetime on the temperature was carried out using streak camera measurements. Consistent with the literature ${ }^{44}$, a significant increase of the carrier lifetime was found when the 
a

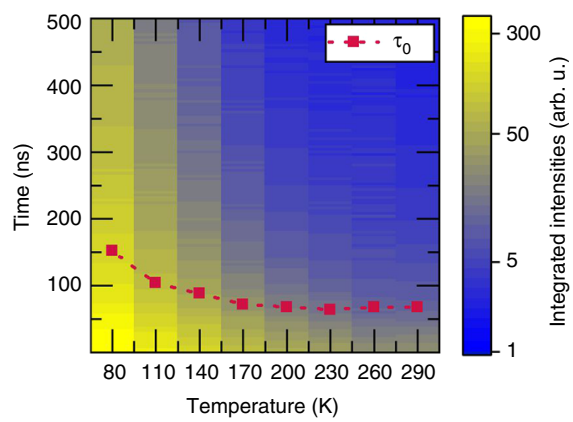

b



Fig. 2 Temperature-dependent PL properties. a Transient PL images showing the integrated intensity as a function of time after fs-excitation and temperature at an excitation fluence of $60 \mathrm{~nJ} \mathrm{~cm}-2$. The red dots denote the carrier lifetime $\tau_{0}$ defined as the time when the integrated intensity dropped to $1 /$ e of its initial intensity. The dotted line is drawn as a guide to the eye. b Peak wavelength and FWHM of the thin film PL. The blue symbols are extracted from the measurement while cooling down and the red symbols are extracted while heating up

temperature was reduced from 290 to $80 \mathrm{~K}$ (Fig. 2a and Supplementary Figure 4). Additionally, the total signal strength at constant excitation fluence increases substantially at lower temperatures due to the typical increase of the photoluminescence quantum yield (PLQY) ${ }^{45,46}$. Consequently, the ratio of radiative to non-radiative recombination increases, leading to a lower ASE onset threshold. In contrast to $\mathrm{CH}_{3} \mathrm{NH}_{3} \mathrm{PbI}_{3}$, where the structural phase change at $160 \mathrm{~K}$ leads to a drastic and sudden change in the PL properties, the PL spectrum in our triple cation material shifts steadily over the entire temperature range without any noticeable hysteresis (Supplementary Figure 5). The PL peak wavelength shifts gradually from 766 to $783 \mathrm{~nm}$ while its FWHM narrows from 46 to $16 \mathrm{~nm}$ as the temperature is decreased from 290 to $80 \mathrm{~K}$ (Fig. 2b), clearly indicating the existence of a single phase across the transition from room to cryogenic temperatures.

ASE under CW excitation. The source used for the CW pumping experiments was a diode-pumped solid-state laser (Millennia, Spectra Physics, $532 \mathrm{~nm}, P_{\max }=3.3 \mathrm{~W}$ ). Figure $3 \mathrm{a}$ depicts the normalized emission spectra at several different $\mathrm{CW}$ pump intensities measured at $80 \mathrm{~K}$. Similar to the pulsed excitation case, the typical ASE shoulder evolves in the spectra between 786 and $794 \mathrm{~nm}$ and develops into a dominating peak. Figure $3 \mathrm{~b}$ depicts a log-scale plot of the luminescence spectra for all recorded pump intensities (Supplementary Figure 6 includes the detailed pump powers and include a linear scale plot, Supplementary Figures 7 and 8 depict the normalized spectra). Above the ASE threshold, most of the additional pump power is fed into ASE when the excitation intensity is increased. Using the above-mentioned approach, the ASE threshold intensity under CW excitation was found to be $I_{\mathrm{th}, \mathrm{CW}}=387 \mathrm{~W} \mathrm{~cm}^{-2}$ (see Fig. 3c). Note that above threshold, the FWHM of the ASE peak collapses rapidly, reaching values of $\sim 2 \mathrm{~nm}$ at high pumping intensities. The clear threshold and the dramatic narrowing of the peak proves the generation of ASE under CW excitation. Figure $2 \mathrm{~d}$ presents a comparison of the emission spectra under CW excitation at low intensity (well below threshold, $6 \mathrm{~W} \mathrm{~cm}^{-2}$ ), at high intensity (well above threshold, $1258 \mathrm{~W} \mathrm{~cm}^{-2}$ ), as well as the corresponding spectra under pulsed excitation above threshold fluence $\left(\sim 14 \mu \mathrm{J} \mathrm{cm}^{-2}\right)$. The spectra at high CW intensities and pulsed excitation are comparable, proving that the material's emission properties and the crystal phase remain unchanged when CW ASE starts. Note that sustaining the same crystal morphology under pulsed and CW excitation constitutes a significant improvement with respect to the previous $\mathrm{CW}$ lasing observations in $\mathrm{CH}_{3} \mathrm{NH}_{3} \mathrm{PbI}_{3}{ }^{32}$, thus demonstrating that $\mathrm{CW}$ stimulated emission can be sustained in single-phase perovskites. The limited thermal conductivity of perovskites does not seem to be a major issue since the ASE peak wavelengths for pulsed and CW excitations are identical.

The CW threshold $I_{\text {th,CW }}$ value is nearly an order of magnitude lower than the corresponding peak intensity at pulsed excitation $\left(I_{\text {th,pulsed }}=2750 \mathrm{~W} \mathrm{~cm}^{-2}\right)$, calculated as the threshold fluence divided by the pump duration $(0.8 \mathrm{~ns})$ under pulsed excitation. This is, however, not surprising as the carrier lifetime at $80 \mathrm{~K}$ is substantially longer than the pump pulse duration. When the excitation pulse is shorter than the carrier lifetime, the threshold power and the pulse energy are rather related by ${ }^{47}$

$$
I_{\text {th, CW }} \cdot \tau=F_{\text {th }}
$$

where $\tau$ is the carrier lifetime. Dividing our observed values of $F_{\text {th }}$ and $I_{\text {th,CW }}$ results in a coarse estimate for the carrier lifetime at low temperature of $5.7 \mathrm{~ns}$, which is entirely reasonable considering our streak camera measurements, even when considering the significant additional Auger processes for higher excitation densities (Fig. 2a).

The plausibility of the ASE threshold can be tested using the Bernard-Duraffourg condition ${ }^{48}$ which, for equal effective masses for both carriers, requires the quasi-Fermi levels for electrons and holes to reach the conduction and valence band energy levels, respectively. Assuming $m_{\mathrm{e}}=m_{\mathrm{h}}$ for perovskite semiconductors ${ }^{3}$ and using standard equations for the carrier density in $3 \mathrm{D}$ semiconductors with parabolic bands, the carrier density at the point when the quasi-Fermi levels reach the band edges can be estimated to be $1.54 \times 10^{17} \mathrm{~cm}^{-3}$ at $80 \mathrm{~K}$ (see Supplementary Note 1 for detailed calculations). Therefore, the minimal carrier lifetime required for reaching a given carrier density at a given excitation rate can be readily estimated (see Supplementary Note 2 for details). Using a generation rate based on the threshold intensity of $387 \mathrm{~W} \mathrm{~cm}^{-2}$, we find that a carrier lifetime of at least $3.9 \mathrm{~ns}$ is required in order to reach (or exceed) a density of $1.54 \times$ $10^{17} \mathrm{~cm}^{-3}$, required for net optical gain. As seen in Fig. $2 \mathrm{a}$, the measured carrier lifetime at low excitations is well above this value.

In addition to low thresholds, lasing stability is an important requirement for any laser gain medium. Figure $4 \mathrm{a}$ shows the integrated emission intensity under constant $\mathrm{CW}$ excitation of $720 \mathrm{~W} \mathrm{~cm}^{-2}$ for a period of $10 \mathrm{~min}$ at a temperature of $80 \mathrm{~K}$. Figure $4 \mathrm{~b}$ depicts the corresponding spectra at the beginning and 
a

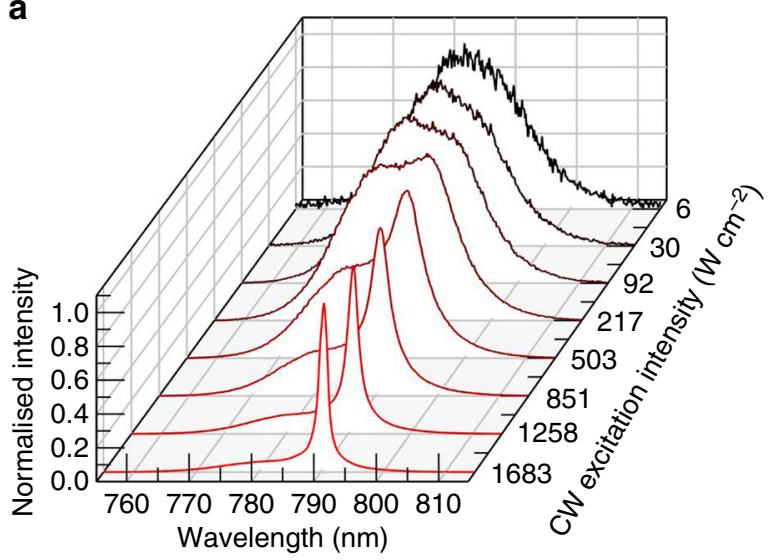

C

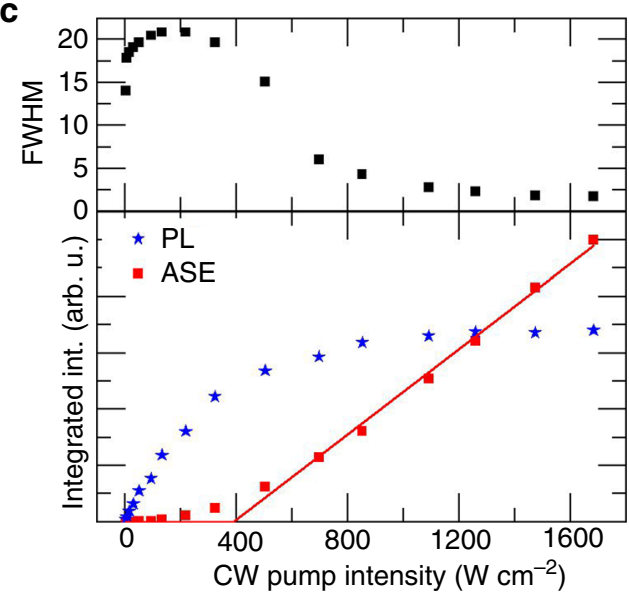

b



d

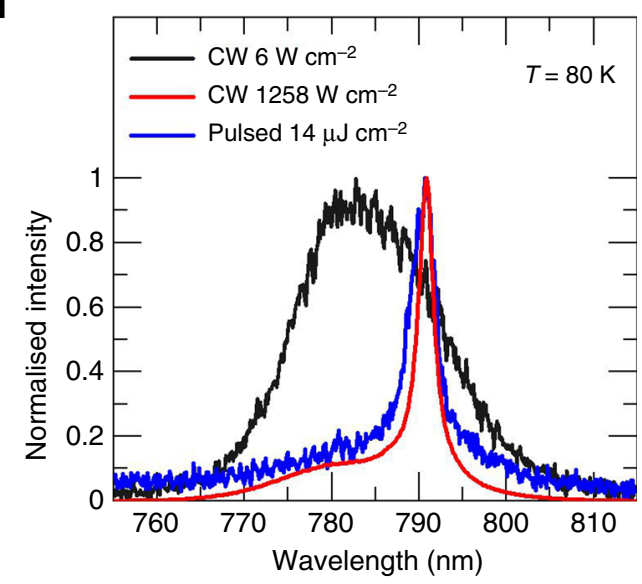

Fig. 3 Characterization of CW ASE at $80 \mathrm{~K}$. a The emission spectrum changes for increasing excitation intensities from a broadband PL spectrum (FWHM 15-20 nm) to a narrowband ASE spectrum (FWHM $\sim 2 \mathrm{~nm}$ ). b Logarithmic representation of the luminescence spectra for increasing excitation intensities showing the rise of ASE between 786 and $794 \mathrm{~nm}$. c PL, ASE, and FWHM as a function of the CW excitation intensity. At the threshold intensity of $387 \mathrm{~W} \mathrm{~cm}^{-2}$, the FWHM decreases significantly and most of the additional pump intensity is fed into the ASE band. $\mathbf{d}$ Comparison of CW PL, CW ASE, and pulsed ASE spectra at $80 \mathrm{~K}$
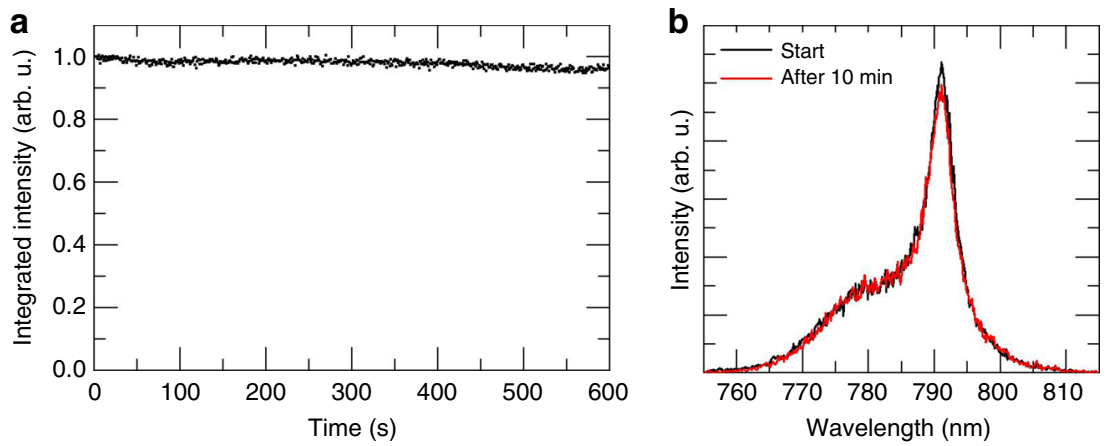

Fig. 4 ASE stability at CW excitation with an excitation intensity of $720 \mathrm{~W} \mathrm{~cm}^{-2}$ at $80 \mathrm{~K}$. a Integrated emission intensity over a time frame of 10 min of continuous excitation. $\mathbf{b}$ Spectra at the beginning of the measurement (black) and after $10 \mathrm{~min}$ (red)

at the end of the measurement. A small decrease of the integrated and peak intensities of the ASE is visible, nevertheless, the stability of the emission is remarkable at such harsh excitation conditions. This demonstrates that the stability under CW excitation is comparable to the stability under pulsed excitation reported previously $11,12,32,49$. Additionally, we note that the wavelength of the ASE peak did not change during the experiment. The absence of such a shift, which is observed in $\mathrm{CH}_{3} \mathrm{NH}_{3} \mathrm{PbX}_{3}$ perovskites due to a redistribution of the halides ${ }^{50}$, confirms the stability of the perovskite crystal lattice with mixed cations at the A-site 33,34

Next, we tracked the emission properties at higher temperatures under fixed CW pumping conditions as shown in Fig. 5. All measurements were carried out at a CW pump intensity of 1512 $\mathrm{W} \mathrm{cm}{ }^{-2}$. As the temperature is increased, the ASE peak decreases and a larger portion of the absorbed photons is reemitted by spontaneous emission (instead of stimulated emission). The ASE peak fades out at temperatures above $120 \mathrm{~K}$. At this temperature 


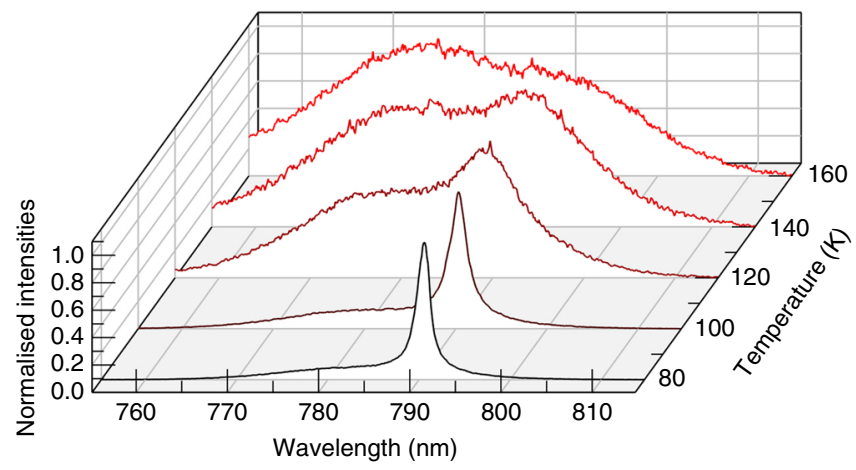

Fig. 5 ASE spectra under CW excitation at various temperatures and constant pump intensity. The amount of photons emitted into the ASE mode decreases with increasing temperature until $120 \mathrm{~K}$, after which spontaneous emission dominates. The excitation power was kept constant at $1512 \mathrm{~W} \mathrm{~cm}^{-2}$

(and above), a further increase of the pump intensity could not recover the ASE peak, but rather led to a fast degradation of the PL intensity. This indicates that at higher temperatures the material degrades before the CW ASE threshold is reached. However, the fact that the ASE peaks can be observed over the full range between 80 and $120 \mathrm{~K}$, is another indication that the ASE is obtained by a single crystalline phase and not due to the coexistence of different phases as in $\mathrm{CH}_{3} \mathrm{NH}_{3} \mathrm{PbI}_{3}$, which requires a very specific temperature ${ }^{32}$.

Several approaches can be employed in order to increase the temperature range where ASE can be achieved before material degradation starts. This includes better thermal management, such as pumping the layer using photons with lower energy, closer to the bandgap, consequently introducing less heating during the thermalization of carriers to the band edges. In addition, an enhancement of the carrier lifetime and the PLQY at higher temperatures by a further improvement of the material quality should also allow for ASE generation at higher temperatures. Furthermore, the use of high-Q cavities can decrease the threshold values for laser devices compared to our ASE threshold in thin films. Our work manifests a crucial step towards the realization of a new class of laser materials, which can potentially support room-temperature CW lasing under optical pumping. In view of the high charge carrier mobilities in our material, this also raises prospects for future electrically pumped devices. Thus, a new class of low-cost and solution processable laser diodes with tunability over the full visible spectrum might be at the horizon.

\footnotetext{
Methods

Materials and sample preparation. The precursor powders $\mathrm{PbI}_{2}$ (AlfaAesar), $\mathrm{PbBr}_{2}$ (AlfaAesar), CsI (Alfa Aesar), FAI (Greatcell Solar Ltd.), and MABr (Greatcell Solar Ltd.) were mixed in a non-stoichiometric ratio of $\mathrm{Cs}_{0.1}\left(\mathrm{MA}_{0.17^{-}}\right.$ $\left.\mathrm{FA}_{0.83}\right)_{0.9} \mathrm{~Pb}_{0.84}\left(\mathrm{I}_{0.84} \mathrm{Br}_{0.16}\right)_{2.68}$ and dissolved in a mixture of dimethylformamide (DMF, Sigma Aldrich) and dimethyl sulfoxide (DMSO, Sigma Aldrich) in a ratio of 3:1. All chemicals and solvents were used as received without further purification. The sapphire substrates ( 1 " diameter, $1 \mathrm{~mm}$ thick, Plano $\mathrm{GmbH}$ ) were cleaned in an ultrasonic bath using acetone and isopropanol for $10 \mathrm{~min}$ each and treated by $\mathrm{O}_{2}$ plasma for $2 \mathrm{~min}$ at $100 \mathrm{~W}$. Directly after the plasma treatment $70 \mu \mathrm{l}$ of the solution were dynamically spin-casted at $1000 \mathrm{rpm}$ for $10 \mathrm{~s}$ and $6000 \mathrm{rpm}$ for $20 \mathrm{~s}$ in nitrogen atmosphere. Six seconds before the end of the spinning process, $160 \mu \mathrm{l}$ of chlorobenzene were dropped onto the spinning sample. The resulting films were annealed at $100{ }^{\circ} \mathrm{C}$ for $60 \mathrm{~min}$ in $\mathrm{N}_{2}$. Afterwards, the films were post treated by imprint lithography. The imprint step used in this work is based on previously published studies ${ }^{35-37}$ and conducted using a commercially available nanoimprint lithography instrument (FC-150, SET) with the following recipe: A glass substrate covered with a thin layer of OrmoStamp ${ }^{\oplus}$ was pressed into the perovskite substrate with a pressure of 10 bar to form an initial contact between the mold and the substrate. This was followed by heating the system to $125^{\circ} \mathrm{C}$, the final imprint temperature. Then, the imprint pressure was raised to 125 bar for a duration of 6
}

min followed by the cooling of the system for a duration of $6.5 \mathrm{~min}$ to achieve a final temperature of $\sim 50^{\circ} \mathrm{C}$.

ASE measurements. A schematic of the gain characterization setup is shown in Supplementary Figure S2: For the experiments under pulsed excitation, a frequency doubled Nd:YLF laser (Innolas $\mathrm{GmbH}$ ), emitting pulses of $0.8 \mathrm{~ns}$ pulse duration at $532 \mathrm{~nm}$ was used. For the CW excitation experiments, a CW laser emitting at the same wavelength was used (Millennia, Spectra Physics). The pump intensities were controlled using variable neutral density filter wheels. The laser power was measured using a Coherent LabMax 2 power meter with various power heads. The samples were mounted in an optical cryostat (Janis STVP-100) and connected via heat-conductive paste to a copper sample holder. The spot area at the sample position inside the cryostat was determined by the moving knife edge method to be $1.28 \times 10^{-4} \mathrm{~cm}^{2}$ for the pulsed beam and $5.01 \times 10^{-4} \mathrm{~cm}^{2}$ for the CW laser beam. The sample emission was coupled through a pair of lenses to a spectrograph (Acton Research Corporation, SpectraPro 300i, variable grating) connected to an intensified CCD camera (Princeton Research, PiMax 512).

Time resolved and temperature-dependent PL. The PL transients in Fig. 2a were recorded by a streak camera (Hamamatsu Universal C10910) coupled to a spectrometer (Acton SpectraPro SP2300). The camera was used in single sweep mode to allow for a $2 \mu$ s time window and a FWHM of the instrumental response function of $28 \mathrm{~ns}$. The steady-state PL spectra shown in Fig. $2 \mathrm{~b}$ were recorded by a fiber-coupled spectrometer (Avantes, AvaSpec-2048L). In both cases, the temperature of the sample was controlled by a closed cycle cryostat (Oxford, Optistat Dry TLEX). For the excitation, a second harmonic generator (Coherent, Chameleon Compact OPO-Vis) was used to double the frequency of a mode-locked Ti:sapphire laser (Coherent, Chameleon Ultra) with a wavelength of $960 \mathrm{~nm}$, a pulse width of $140 \mathrm{fs}$ and a repetition rate of $80 \mathrm{MHz}$. The repetition rate of the laser system was reduced to $400 \mathrm{kHz}$ using a pulse picker (APE, pulseSelect).

\section{Data availability}

The data that support the findings of this study are available from the corresponding author upon reasonable request.

Received: 31 October 2018 Accepted: 5 February 2019

Published online: 28 February 2019

\section{References}

1. Zhang, W., Eperon, G. E. \& Snaith, H. J. Metal halide perovskites for energy applications. Nat. Energy 1, 16048 (2016).

2. Correa-Baena, J.-P. et al. Promises and challenges of perovskite solar cells. Science 358, 739-744 (2017).

3. Herz, L. M. Charge-carrier mobilities in metal halide Perovskites: fundamental mechanisms and limits. ACS Energy Lett. 2, 1539-1548 (2017).

4. Brenes, R. et al. Metal halide Perovskite polycrystalline films exhibiting properties of single crystals. Joule 1, 155-167 (2017).

5. Colella, S., Mazzeo, M., Rizzo, A., Gigli, G. \& Listorti, A. The bright side of Perovskites. J. Phys. Chem. Lett. 7, 4322-4334 (2016).

6. Sutherland, B. R. \& Sargent, E. H. Perovskite photonic sources. Nat. Photonics 10, 295-302 (2016)

7. Cho, H. et al. Overcoming the electroluminescence efficiency limitations of perovskite light-emitting diodes. Science 350, 1222-1225 (2015).

8. Tan, Z.-K. et al. Bright light-emitting diodes based on organometal halide perovskite. Nat. Nanotechnol. 9, 1-6 (2014).

9. Lin, K. et al. Perovskite light-emitting diodes with external quantum efficiency exceeding 20 per cent. Nature 562, 245-248 (2018).

10. Kovalenko, M. V., Protesescu, L. \& Bodnarchuk, M. I. Properties and potential optoelectronic applications of lead halide perovskite nanocrystals. Science 358, 745-750 (2017)

11. Xing, G. et al. Low-temperature solution-processed wavelength-tunable perovskites for lasing. Nat. Mater. 13, 476-480 (2014).

12. Brenner, P. et al. Triple cation mixed-halide perovskites for tunable lasers. Opt. Mater. Express 7, 4082 (2017).

13. Zhang, Q. et al. Advances in small Perovskite-based lasers. Small Methods 1, 1700163 (2017).

14. Suárez Alvarez, I. Active photonic devices based on colloidal semiconductor nanocrystals and organometallic halide perovskites. Eur. Phys. J. Appl. Phys. 75, 30001 (2016).

15. Wang, K., Wang, S., Xiao, S. \& Song, Q. Recent advances in Perovskite microand nanolasers. Adv. Opt. Mater. 6, 1800278 (2018).

16. Li, G., Price, M. \& Deschler, F. Research update: challenges for high-efficiency hybrid lead-halide perovskite LEDs and the path towards electrically pumped lasing. APL Mater. 4, 091507 (2016). 
17. Veldhuis, S. A. et al. Perovskite materials for light-emitting diodes and lasers. Adv. Mater. 28, 6804-6834 (2016).

18. Quan, L. N., García de Arquer, F. P., Sabatini, R. P. \& Sargent, E. H. Perovskites for light emission. Adv. Mater. 30, 1801996 (2018).

19. Harwell, J. R., Whitworth, G. L., Turnbull, G. A. \& Samuel, I. D. W. Green Perovskite distributed feedback lasers. Sci. Rep. 7, 11727 (2017).

20. Chen, S. \& Nurmikko, A. Stable green Perovskite vertical-cavity surface-emitting lasers on rigid and flexible substrates. ACS Photonics 4, 2486-2494 (2017).

21. Pust, P., Schmidt, P. J. \& Schnick, W. A revolution in lighting. Nat. Mater. 14, 454-458 (2015).

22. Auf Der Maur, M., Pecchia, A., Penazzi, G., Rodrigues, W. \& Di Carlo, A. . Efficiency drop in green InGaN/GaN light emitting diodes: the role of random alloy fluctuations. Phys. Rev. Lett. 116, 1-5 (2016).

23. Strauss, U. et al. in GaInN laser diodes from 440 to $530 \mathrm{~nm}$ : a performance study on single-mode and multi-mode R\&D designs, Proc. SPIE vol. 10123 (eds. Belyanin, A. A. \& Smowton, P. M.) 101230A (International Society for Optics and Photonics, 2017).

24. Fan, F. et al. Continuous-wave lasing in colloidal quantum dot solids enabled by facet-selective epitaxy. Nature 544, 75-79 (2017).

25. Sandanayaka, A. S. D. et al. Toward continuous-wave operation of organic semiconductor lasers. Sci. Adv. 3, e1602570 (2017).

26. Grim, J. Q. et al. Continuous-wave biexciton lasing at room temperature using solution-processed quantum wells. Nat. Nanotechnol. 9, 891-895 (2014).

27. Yang, Z., Pelton, M., Fedin, I., Talapin, D. V. \& Waks, E. A room temperature continuous-wave nanolaser using colloidal quantum wells. Nat. Commun. 8, 143 (2017)

28. Evans, T. J. S. et al. Continuous-wave lasing in cesium lead bromide perovskite nanowires. Adv. Opt. Mater. 6, 1-7 (2018)

29. Su, R. et al. Room-temperature polariton lasing in all-inorganic Perovskite nanoplatelets. Nano Lett. 17, 3982-3988 (2017)

30. Jia, Y. et al. Diode-pumped organo-lead halide perovskite lasing in a metalclad distributed feedback resonator. Nano Lett. 16, 4624-4629 (2016).

31. Cadelano, M. et al. Can trihalide lead Perovskites support continuous wave lasing? Adv. Opt. Mater. 3, 1557-1564 (2015).

32. Jia, Y., Kerner, R. A., Grede, A. J., Rand, B. P. \& Giebink, N. C. Continuouswave lasing in an organic-inorganic lead halide perovskite semiconductor. Nat. Photonics 11, 784-788 (2017).

33. Saliba, M. et al. Cesium-containing triple cation perovskite solar cells: improved stability, reproducibility and high efficiency. Energy Environ. Sci. 9, 1989-1997 (2016).

34. McMeekin, D. P. et al. A mixed-cation lead mixed-halide perovskite absorber for tandem solar cells. Science 351, 151-155 (2016).

35. Pourdavoud, N. et al. Photonic nanostructures patterned by thermal nanoimprint directly into organo-metal halide Perovskites. Adv. Mater. 29, 1605003 (2017).

36. Mayer, A. et al. Thermal nanoimprint to improve the morphology of MAPbX3 (MA=methylammonium, $\mathrm{X}=\mathrm{I}$ or Br). J. Vac. Sci. Technol. B 35, 06 G803 (2017)

37. Bar-On, O., Brenner, P., Lemmer, U. \& Scheuer, J. Micro lasers by scalable lithography of metal-halide perovskites. Adv. Mater. Technol. 3, 1800212 (2018).

38. Qin, L. et al. Enhanced amplified spontaneous emission from morphologycontrolled organic-inorganic halide perovskite films. RSC $A d v .5$, 103674-103679 (2015).

39. Siegman, A. E. Lasers. (Univ. Science Books, Sausalito, CA, USA, 1986).

40. Samuel, I. D. W., Namdas, E. B. \& Turnbull, Ga. How to recognize lasing. Nat. Photonics 3, 546-549 (2009).

41. Ning, C. Z. What is laser threshold? IEEE J. Sel. Top. Quantum Electron. 19, 1503604-1503604 (2013).

42. Sutherland, B. R., Hoogland, S., Adachi, M. M., Wong, C. T. O. \& Sargent, E. $\mathrm{H}$. Conformal organohalide perovskites enable lasing on spherical resonators. ACS Nano 8, 10947-10952 (2014).

43. Coldren, L. A., Corzine, S. W. \& Mašanović, M. L. Diode Lasers and Photonic Integrated Circuits. Series in Microwave and Optical Engineering. (John Wiley \& Sons, Inc., Hoboken, NJ, USA, 2012).

44. Milot, R. L., Eperon, G. E., Snaith, H. J., Johnston, M. B. \& Herz, L. M. Temperature-dependent charge-carrier dynamics in $\mathrm{CH}_{3} \mathrm{NH}_{3} \mathrm{PbI}_{3}$ Perovskite thin films. Adv. Funct. Mater. 25, 6218-6227 (2015).
45. Stranks, S. D. et al. Recombination kinetics in organic-inorganic Perovskites: excitons, free charge, and subgap states. Phys. Rev. Appl. 2, 1-8 (2014).

46. Braly, I. L. et al. Hybrid perovskite films approaching the radiative limit with over $90 \%$ photoluminescence quantum efficiency. Nat. Photonics 12, 355-361 (2018).

47. Noginov, M. A., Fowlkes, I. N., Zhu, G. \& Novak, J. Random laser thresholds in $\mathrm{cw}$ and pulsed regimes. Phys. Rev. A 70, 043811 (2004)

48. Bernard, M. G. A. \& Duraffourg, G. Laser conditions in semiconductors. Phys. Status Solidi 1, 699-703 (1961).

49. Brenner, P. et al. Highly stable solution processed metal-halide perovskite lasers on nanoimprinted distributed feedback structures. Appl. Phys. Lett. 109, 141106 (2016).

50. Hoke, E. T. et al. Reversible photo-induced trap formation in mixed-halide hybrid perovskites for photovoltaics. Chem. Sci. 6, 613-617 (2015)

\section{Acknowledgements}

The authors are grateful to the all members of the perovskite taskforce at KIT. Funding is gratefully acknowledged from the following sources: (i) the Deutsche Forschungsgemeinschaft via project LE 878/17-1; (ii) the Helmholtz Research Program Science and Technology of Nanosystems (STN), the Initiating and Networking funds of the Helmholtz Association (HYIG of Dr. U.W. Paetzold, Recruitment Initiative of Prof B.S. Richards, the Helmholtz Energy Materials Foundry (HEMF); and PEROSEED project); (iii) the Karlsruhe School of Optics \& Photonics (KSOP) as well as (iv) the 373 Excellence Cluster "3D Matter Made to Order (3DMM2O)".

\section{Author contributions}

The project was designed and conceptualized by P.B., U.W.P, I.A.H. and U.L. P.B. performed the ASE and AFM measurements, analyzed data and wrote the first draft of the paper. O.B.-O. performed imprinting of perovskites films and discussed the results. M.J. performed time-resolved measurements and temperature-dependent PL spectroscopy. I.A.H. assisted in the experimental work. B.S.R., U.W.P., I.A.H, J.S., and U.L. supervised the overall conception. All authors contributed to the discussion of the results and in writing the manuscript.

\section{Additional information}

Supplementary Information accompanies this paper at https://doi.org/10.1038/s41467 019-08929-0.

Competing interests: The authors declare no competing interests.

Reprints and permission information is available online at http://npg.nature.com/ reprintsandpermissions/

Journal peer review information: Nature Communications thanks the anonymous reviewers for their contribution to the peer review of this work.

Publisher's note: Springer Nature remains neutral with regard to jurisdictional claims in published maps and institutional affiliations.

Open Access This article is licensed under a Creative Commons Attribution 4.0 International License, which permits use, sharing, adaptation, distribution and reproduction in any medium or format, as long as you give appropriate credit to the original author(s) and the source, provide a link to the Creative Commons license, and indicate if changes were made. The images or other third party material in this article are included in the article's Creative Commons license, unless indicated otherwise in a credit line to the material. If material is not included in the article's Creative Commons license and your intended use is not permitted by statutory regulation or exceeds the permitted use, you will need to obtain permission directly from the copyright holder. To view a copy of this license, visit http://creativecommons.org/ licenses/by/4.0/

(C) The Author(s) 2019 